\title{
Non-convex Representations of Graphs ${ }^{\star}$
}

\author{
Giuseppe Di Battista, Fabrizio Frati, and Maurizio Patrignani \\ Dip. di Informatica e Automazione - Roma Tre University
}

\begin{abstract}
We show that every plane graph admits a planar straight-line drawing in which all faces with more than three vertices are non-convex polygons.
\end{abstract}

\section{Introduction}

In a straight-line planar drawing of a graph each edge is drawn as a segment and no two segments intersect. A convex drawing is a straight-line planar drawing in which each face is a convex polygon. While every plane graph admits a planar straight-line drawing [6], not every plane graph admits a convex drawing. Tutte showed that every triconnected plane graph admits such a drawing with its outer face drawn as an arbitrary convex polygon [12]. Thomassen [11] characterized the graphs admitting a convex drawing and Chiba et al. [3] presented a linear-time algorithm for producing such drawings. Convex drawings can be efficiently constructed in small area [4]2].

Hong and Nagamochi proved that for every triconnected plane graph whose boundary is a star-shaped polygon a drawing in which every internal face is a convex polygon can be obtained in linear time [8]. The same authors also investigated drawings where the outer face is a convex polygon and the internal faces are star-shaped [9 10].

As opposed to traditional convex graph drawing, some research works explored the properties of drawings with non-convexity requirements [7|1]. A pointed drawing is such that each vertex is incident to an angle larger than $\pi$. In [7] it is shown that a planar graph admits a straight-line pointed drawing iff it is minimally rigid or a subgraph of a minimally rigid graph. Since there exist planar graphs that do not admit straight-line pointed drawings, algorithms that construct pointed drawings with tangent-continuous biarcs, circular arcs, or parabolic arcs are studied in [1].

In this paper we address the problem of producing non-convex drawings, i.e., drawings where all faces with more than three vertices are non-convex. This can be considered as the opposite of the classic problem of constructing convex drawings. Also, it can be seen as the dual of the problem of constructing pointed drawings, since faces, and not vertices, are constrained to have an angle greater than $\pi$. We prove the following:

Theorem 1. Every plane graph admits a non-convex drawing.

In Sect. 3 we prove the previous theorem for biconnected graphs by means of a constructive algorithm whose inductive approach is reminescent of Fary's construction [6], although it applies to non-triangulated graphs and relies on a more complex case study. Due to space restrictions, some proofs are omitted and can be found in [5]. In Sect. 4 we discuss how to extend the result to general plane graphs.

\footnotetext{
* Work partially supported by MUR under Project "MAINSTREAM: Algoritmi per strutture informative di grandi dimensioni e data streams."
} 


\section{Preliminaries}

A graph $G$ is simple if it has no multiple edges and no self-loops. A planar drawing of $G$ determines a circular ordering of the edges incident to each vertex. Two drawings of $G$ are equivalent if they determine the same circular ordering around each vertex. A planar embedding is an equivalence class of planar drawings. A planar drawing partitions the plane into faces. The unbounded face is the outer face and is denoted by $f(G)$. A chord of $f(G)$ is an edge connecting two non-adjacent vertices of $f(G)$. A graph with a planar embedding and an outer face is called a plane graph. A non-convex drawing of a plane graph $G$ is a planar straight-line drawing of $G$ in which each internal (external) face with more than three vertices has an angle greater than $\pi$ (smaller than $\pi$ ).

Consider a face $f$ of $G$ and two non-adjacent vertices $u$ and $v$ incident to $f$. The contraction of $u$ and $v$ inside $f$ leads to a graph $G^{\prime}$ in which $u$ and $v$ are replaced by a single vertex $w$, connected to all vertices $u$ and $v$ are connected to in $G$. If $u$ and $v$ are connected to the same vertex $y$, then $G^{\prime}$ contains two edges $(w, y)$ (hence $G^{\prime}$ is not simple), unless $(u, y)$ and $(y, v)$ are incident to $f$ (in this case there is only one edge $(w, y))$. A contraction can also be performed on two adjacent vertices $u$ and $v$, by removing edge $(u, v)$ and contracting $u$ and $v$ inside the face created by the removal.

\section{Proof of Theorem 1}

In this section we assume that graph $G$ is biconnected. The following lemmata hold.

Lemma 1. Suppose that $G$ has a face $f$ with at least four incident vertices. Then there exist two vertices $u$ and $v$ incident to $f$, such that edge $(u, v)$ can be added to $G$ inside $f$, so that the resulting plane graph $G^{\prime}$ is simple.

Lemma 2. Let $G$ be a plane graph with a face $f$ having more than four incident vertices. Let $G^{\prime}$ be the plane graph obtained by inserting inside $f$ an edge e between two non-adjacent vertices of $f$. Suppose that a non-convex drawing $\Gamma^{\prime}$ of $G^{\prime}$ exists. Then the drawing $\Gamma$ obtained by removing e from $\Gamma^{\prime}$ is a non-convex drawing of $G$.

In order to prove Theorem 1 for biconnected plane graphs, we prove the following:

Theorem 2. Let $G$ be a biconnected plane graph such that all the faces of $G$ have three or four incident vertices. Let $f(G)$ be the outer face of $G$. If $f(G)$ has three vertices, then, for every triangle $T$ in the plane, $G$ admits a non-convex drawing in which $f(G)$ is represented by $T$. If $f(G)$ has four vertices and no chord, then, for every non-convex quadrilateral $Q$ in the plane, $G$ admits a non-convex drawing in which $f(G)$ is represented by $Q$. The mapping of the vertices of $f(G)$ to the vertices of $T$ or $Q$ is arbitrary, provided that the circular ordering of the vertices of $f(G)$ is respected.

Theorem 2 together with Lemmata 1 and 2 implies Theorem 1 Namely, let $G$ be any biconnected plane graph. While $G$ has any face $f$ with at least five vertices, add, by Lemma 1 a dummy edge inside $f$ so that the augmented graph is still plane and simple. The obtained graph $G^{\prime}$ has (at least) one face with four incident vertices for each face of $G$ with more than three incident vertices. In order to apply Theorem 2 , consider 


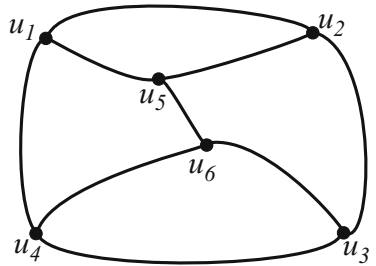

(a)

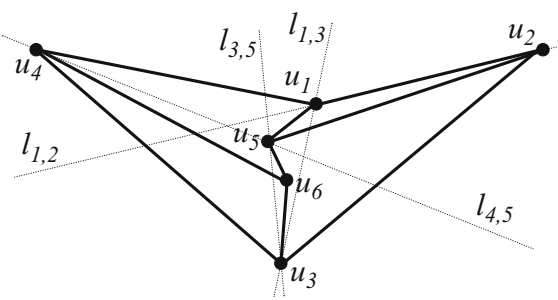

(b)

Fig. 1. (a) $G_{6}^{*}$. (b) Construction of a non-convex drawing of $G_{6}^{*}$.

$f\left(G^{\prime}\right)$ and, if $f\left(G^{\prime}\right)$ has four incident vertices and has a chord, insert a dummy edge inside $f\left(G^{\prime}\right)$ between the two vertices not incident to the chord, turning $f\left(G^{\prime}\right)$ into a triangular face. Construct a non-convex drawing of $G^{\prime}$, as in the proof of Theorem 2 By Lemma 2, removing the dummy edges inserted leaves the drawing non-convex.

Before proving Theorem 2 we give two more lemmata. Let $G_{5}^{*}$ be the plane graph having a 4 -cycle $\left(u_{1}, u_{2}, u_{3}, u_{4}\right)$ as outer face and one internal vertex $u_{5}$ connected to $u_{1}, u_{2}, u_{3}$, and $u_{4}$. Let $G_{6}^{*}$ be the plane graph having a 4 -cycle $\left(u_{1}, u_{2}, u_{3}, u_{4}\right)$ as outer face and two connected internal vertices $u_{5}$ and $u_{6}$ with $u_{5}$ connected to $u_{1}$ and $u_{2}$, and $u_{6}$ connected to $u_{3}$ and $u_{4}$ (see Fig. 1 a).

Lemma 3. For any non-convex quadrilateral $Q$ in the plane, there exists a non-convex drawing of $G_{5}^{*}$ such that $f\left(G_{5}^{*}\right)$ is represented by $Q$.

Lemma 4. For any non-convex quadrilateral $Q$ in the plane, there exists a non-convex drawing of $G_{6}^{*}$ such that $f\left(G_{6}^{*}\right)$ is represented by $Q$ (see Fig. 1 b).

Proof of Theorem 2. Let $G$ be a biconnected plane graph having all faces of size three or four and such that, if $f(G)$ has size four, then it has no chord.

The proof is by induction on the number of internal vertices of $G$. In the base case, either $G$ has no internal vertex and the statement is trivially true, or $G=G_{5}^{*}$, or $G=G_{6}^{*}$. In the latter cases the statement follows by Lemmata 3 and 4 , respectively. Inductively assume that the statement holds for any biconnected plane graph with less than $n$ internal vertices. Suppose that $G$ has $n$ internal vertices. Three are the cases.

$\boldsymbol{G}$ has a separating 3 -cycle $\boldsymbol{C}$. Denote by $G_{1}$ the graph obtained by removing from $G$ all vertices internal to $C$. Denote by $G_{2}$ the subgraph of $G$ induced by the vertices internal to and on the border of $C$. If $f\left(G_{1}\right)$ has three (four) vertices, then consider any triangle $T$ (resp. any non-convex quadrilateral $Q$ ) and construct a non-convex drawing $\Gamma_{1}$ of $G_{1}$ having $T$ (resp. $Q$ ) as outer face. Consider the triangle $T^{\prime}$ representing $C$ in $\Gamma_{1}$. Construct a non-convex drawing $\Gamma_{2}$ of $G_{2}$ having $T^{\prime}$ as outer face and insert $\Gamma_{2}$ inside $\Gamma_{1}$ by gluing the two drawings along the common face $C$ represented by $T^{\prime}$ in both drawings. The resulting drawing $\Gamma$ is a non-convex drawing of $G$.

$\boldsymbol{G}$ has no separating 3 -cycle and $\boldsymbol{G}$ has a separating 4 -cycle $\boldsymbol{C}$. Denote by $G_{1}$ the graph obtained by removing from $G$ all vertices internal to $C$. Denote by $G_{2}$ the subgraph of $G$ induced by the vertices internal to and on the border of $C$. Notice that $f\left(G_{2}\right)$ has no chords, otherwise $G_{2}$ (and then $G$ ) would have a separating triangle. If 


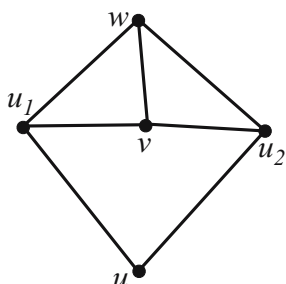

(a)



(b)



(c)

Fig. 2. (a) Both $u_{1}$ and $u_{2}$ are incident to $f(G)$. (b) Both $u_{1}$ and $u_{2}$ are internal vertices of $G$. (c) Vertex $u_{1}$ is incident to $f(G)$ and vertex $u_{2}$ is not.

$f\left(G_{1}\right)$ has three (four) vertices, then consider any triangle $T$ (resp. any non-convex quadrilateral $Q$ ) and construct a non-convex drawing $\Gamma_{1}$ of $G_{1}$ having $T$ (resp. $Q$ ) as outer face. Consider the non-convex quadrilateral $Q^{\prime}$ representing $C$ in $\Gamma_{1}$. Construct a non-convex drawing $\Gamma_{2}$ of $G_{2}$ having $Q^{\prime}$ as outer face and insert $\Gamma_{2}$ inside $\Gamma_{1}$ by gluing the two drawings along the common face $C$ represented by $Q^{\prime}$ in both drawings. The resulting drawing $\Gamma$ is a non-convex drawing of $G$.

$\boldsymbol{G}$ has no separating 3 -cycle and no separating 4-cycle. We are going to contract two vertices of $G$ to obtain a graph with $n-1$ vertices in order to apply induction.

Consider any internal vertex $v$ of $G$. First, we show that at least one of the following statements holds: (i) there exist two triangular faces incident to $v$ and sharing an edge $(v, u)$, such that contracting edge $(v, u)$ does not create chords of $f(G)$; (ii) there exists a quadrilateral face $f=\left(v, u_{1}, u, u_{2}\right)$, such that either contracting $v$ and $u$ or contracting $u_{1}$ and $u_{2}$ does not create chords of $f(G)$; (iii) $G$ is $G_{5}^{*}$; (iv) $G$ is $G_{6}^{*}$.

First, suppose that two triangular faces $\left(u, v, u_{1}\right)$ and $\left(u, v, u_{2}\right)$ exist. If also $u$ is internal to $G$, then contracting $(u, v)$ does not create chords of $f(G)$ and statement (i) holds. Otherwise, assume that $u$ is incident to $f(G)$. Then, statement (i) does not hold only if both the following are true: (a) $f(G)$ is a 4-cycle and (b) there exists an edge connecting $v$ and the vertex $w$ of $f(G)$ not adjacent to $u$. In fact, if (a) does not hold, then $f(G)$ is a 3 -cycle and no chord can be generated by contracting any two vertices. Also, if (b) does not hold, then contracting $v$ to $u$ does not create chords of $f(G)$.

Then, suppose that $f(G)$ is a 4 -cycle $\left(u, w_{1}, w, w_{2}\right)$ and that edge $(v, w)$ exists. If at least one of $u_{1}$ and $u_{2}$ is internal to $G$, then either $\left(u, v, w, w_{1}\right)$ or $\left(u, v, w, w_{2}\right)$ is a separating 4-cycle, contradicting the hypotheses. Hence, assume that both $u_{1}$ and $u_{2}$ are incident to $f(G)$. The four 3 -cycles $\left(u, v, u_{1}\right),\left(u, v, u_{2}\right),\left(w, v, u_{1}\right)$, and $\left(w, v, u_{2}\right)$ do not have internal vertices by hypothesis. Hence, $G$ contains no internal vertex other than $v, G=G_{5}^{*}$, and statement (iii) holds.

If two triangular faces incident to $v$ and sharing an edge do no exist, then there exists a face $f=\left(v, u_{1}, u, u_{2}\right)$. Edge $(u, v)$ does not belong to $G$, otherwise either $\left(v, u, u_{1}\right)$ or $\left(v, u, u_{2}\right)$ would be a separating 3 -cycle. Analogously, edge $\left(u_{1}, u_{2}\right)$ does not belong to $G$. If $u$ is internal to $G$, then contracting $v$ and $u$ in $f$ does not create chords of $f(G)$ and statement (ii) holds. Otherwise, with similar arguments as above, statement (ii) does not hold for $v$ and $u$ only if both the following are true: (a) $f(G)$ is a 4-cycle and (b) there exists an edge connecting $v$ and the vertex $w$ of $f(G)$ not adjacent to $u$. 


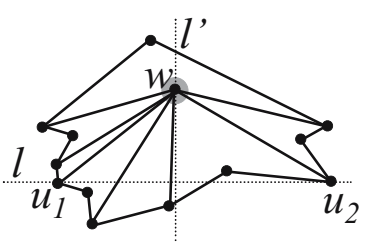

(a)

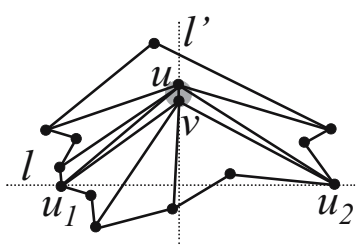

(b)

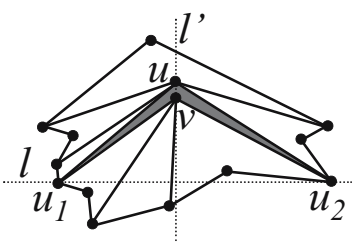

(c)

Fig. 3. (a) and (b): Inductive construction of $\Gamma$, when statement (i) holds. The light shaded region represents disk $D$. (a) and (c): Inductive construction of $\Gamma$, when statement (ii) holds. The dark shaded region represents face $\left(u, u_{1}, v, u_{2}\right)$ in $\Gamma$.

Then, suppose that $f(G)$ is a 4 -cycle and that edge $(v, w)$ exists. We distinguish three cases: (1) If both $u_{1}$ and $u_{2}$ are incident to $f(G)$ (see Fig. 2]a), then $G$ contains 3 -cycles $\left(u_{1}, v, w\right)$ and $\left(u_{2}, v, w\right)$. Since $G$ has no separating triangle, such cycles are faces of $G$, contradicting the hypothesis that two triangular faces incident to $v$ and sharing an edge do no exist. (2) If both $u_{1}$ and $u_{2}$ are internal vertices of $G$ (see Fig. 2]b), then contracting $u_{1}$ and $u_{2}$ does not create chords of $f(G)$ and statement (ii) holds. (3) If one out of $u_{1}$ and $u_{2}$, say $u_{1}$, is incident to $f(G)$ and the other, say $u_{2}$, is not (see Fig. 2]c), consider the fourth vertex $z$ of $f(G)$. If edge $\left(u_{2}, z\right)$ does not exist, vertices $u_{1}$ and $u_{2}$ can be contracted without creating chords of $f(G)$ and statement (ii) holds. If edge $\left(u_{2}, z\right)$ exists, there exist 3 -cycles $\left(u_{1}, v, w\right)$ and $\left(u, u_{2}, z\right)$, and 4-cycles $\left(v, u_{1}, u, u_{2}\right)$ and $\left(v, w, z, u_{2}\right)$. Such cycles contain no vertices in their interior, since $G$ has no separating 3-cycle or 4-cycle. Hence, $G=G_{6}^{*}$ and statement (iv) holds.

We prove that whichever of the statements holds, a non-convex drawing of $G$ can be constructed. Suppose that statement (i) holds, with $G$ having faces $\left(v, u, w_{1}\right)$ and $\left(v, u, w_{2}\right)$. Contract edge $(u, v)$ to a vertex $w$. The resulting graph $G^{\prime}$ is simple. If not, then there would exist a vertex $w_{3}$ adjacent to both $u$ and $v$ in $G$, with $w_{3} \neq w_{1}, w_{2}$. This would imply that $\left(u, v, w_{3}\right)$ is a separating triangle, contradicting the hypotheses.

Inductively construct a non-convex drawing $\Gamma^{\prime}$ of $G^{\prime}$ (see Fig. 3. a). Now consider the point $p$ where vertex $w$ is drawn in $\Gamma^{\prime}$. There exists a small disk $D$ (see Fig. 3 b) centered at $p$ such that moving $p$ to any point inside $D$ leaves $\Gamma^{\prime}$ a non-convex drawing. Consider the line $l^{\prime}$ through $p$ and orthogonal to the line $l$ connecting $u_{1}$ and $u_{2}$. Remove $w$ and its incident edges. Insert vertices $u$ and $v$ on $l^{\prime}$, so that both are inside $D$. Connect $u$ and $v$ to their neighbors. It is easy to see that all faces that had an angle greater than $\pi$ in $\Gamma^{\prime}$ still have an angle greater than $\pi$ in $\Gamma$ and that the drawing is still planar.

Now, suppose that statement (ii) holds. More precisely, suppose that contracting $u$ and $v$ inside a face $f=\left(u, u_{1}, v, u_{2}\right)$ does not create chords of $f(G)$. Contract $u$ and $v$ inside $f$ to a vertex $w$. The resulting graph $G^{\prime}$ is simple. If not, then there would exist a vertex $w_{3}$ adjacent to both $u$ and $v$ in $G$, with $w_{3} \neq u_{1}, u_{2}$. This would imply that either $\left(u, v, u_{1}, w_{3}\right)$ or $\left(u, v, u_{2}, w_{3}\right)$ is a separating 4 -cycle, contradicting the hypotheses.

Inductively construct a non-convex drawing $\Gamma^{\prime}$ of $G^{\prime}$ (see Fig. 3. a). Perturb the vertices so that they are in general position and that $\Gamma^{\prime}$ is still a non-convex drawing. Consider the point $p$ where $w$ is drawn in $\Gamma^{\prime}$ and consider the line $l^{\prime}$ through $p$ and orthogonal to the line $l$ connecting $u_{1}$ and $u_{2}$. There exists a small disk $D$ (see Fig. 3. c) centered at $p$ such that $D$ does not intersect $l$ and such that moving $p$ to any point inside 
$D$ leaves $\Gamma^{\prime}$ non-convex. Remove $w$ and its incident edges. Insert vertices $u$ and $v$ on $l^{\prime}$, so that both are inside $D$. Connect $u$ and $v$ to their neighbors. It is easy to see that all faces that had an angle greater than $\pi$ in $\Gamma^{\prime}$ still have an angle greater than $\pi$ in $\Gamma$ and that the drawing is still planar. Further, face $\left(u, v, u_{1}, u_{2}\right)$ is non-convex, as well, since the angle incident to the one of $u$ and $v$ farther from $l$ is greater than $\pi$.

Finally, suppose that statement (iii) or (iv) holds. We are in one of the base cases and the claim directly follows from Lemmata 3 or 4 respectively.

\section{Conclusions}

We have proved that every biconnected plane graph admits a drawing in which each face with more than three vertices has an angle greater than $\pi$. This result can be extended to general plane graphs as follows. Any simply-connected graph $G$ can be suitably augmented to a biconnected graph $G^{\prime}$ by adding dummy edges in such a way that for each face of $G$ with more than three vertices there exists a corresponding face of $G^{\prime}$ with more than three vertices. Removing dummy edges from a non-convex drawing of $G^{\prime}$ yields a non-convex drawing of $G$. We believe it is of interest to determine whether non-convex drawings can be realized on a polynomial-size grid.

\section{References}

1. Aichholzer, O., Rote, G., Schulz, A., Vogtenhuber, B.: Pointed drawings of planar graphs. In: Bose, P. (ed.) CCCG 2007, pp. 237-240 (2007)

2. Bárány, I., Rote, G.: Strictly convex drawings of planar graphs. Doc. Math. 11, 369-391 (2006)

3. Chiba, N., Yamanouchi, T., Nishizeki, T.: Linear algorithms for convex drawings of planar graphs. In: Bondy, J.A., Murty, U.S.R. (eds.) Progress in Graph Theory, pp. 153-173. Academic Press, New York (1984)

4. Chrobak, M., Goodrich, M.T., Tamassia, R.: Convex drawings of graphs in two and three dimensions. In: Symposium on Computational Geometry, pp. 319-328 (1996)

5. Di Battista, G., Frati, F., Patrignani, M.: Non-convex representations of graphs. Tech. Report RT-DIA-134-2008, Dip. Informatica e Automazione, Univ. Roma Tre (2008)

6. Fary, I.: On straight line representations of planar graphs. Acta. Sci. Math. 11, 229-233 (1948)

7. Haas, R., Orden, D., Rote, G., Santos, F., Servatius, B., Servatius, H., Souvaine, D., Streinu, I., Whiteley, W.: Planar minimally rigid graphs and pseudo-triangulations. Comput. Geometry Theory Appl. 31, 31-61 (2005)

8. Hong, S., Nagamochi, H.: Convex drawings of graphs with non-convex boundary. In: Fomin, F.V. (ed.) WG 2006. LNCS, vol. 4271, pp. 113-124. Springer, Heidelberg (2006)

9. Hong, S., Nagamochi, H.: Star-shaped drawings of planar graphs. In: Brankovich, L., Lin, Y., Smyth, W.F. (eds.) IWOCA 2007. College Publications (2007)

10. Hong, S., Nagamochi, H.: Star-shaped drawing of planar graphs with fixed embedding and concave corner constraints. In: Hu, X., Wang, J. (eds.) COCOON 2008. LNCS, vol. 5092, pp. 405-414. Springer, Heidelberg (2008)

11. Thomassen, C.: Plane representations of graphs. In: Progress in Graph Theory, pp. 43-69. Academic Press, London (1984)

12. Tutte, W.T.: Convex representations of graphs. Proc. London Math. Soc. 10, 304-320 (1960) 